\title{
The Influence of Window Length Analysis on the Time and Frequency Domain of Mechanomyographic and Electromyographic Signals of Submaximal Fatiguing Contractions
}

\author{
Guilherme Nogueira-Neto $^{1,2}$, Eduardo Scheeren ${ }^{2,3}$, Eddy Krueger $^{3}$, Percy Nohama $^{1,2,3}$, \\ Vera Lúcia S. N. Button ${ }^{1}$ \\ ${ }^{1}$ Departamento de Engenharia Biomédica/CEB, Universidade Estadual de Campinas-UNICAMP, Campinas, Brazil \\ ${ }^{2}$ Laboratório de Engenharia de Reabilitação, Pontifícia Universidade Católica do Paraná-PUCPR, Curitiba, Brazil \\ ${ }^{3}$ CPGEI, Universidade Tecnológica Federal do Paraná-UTFPR, Curitiba, Brazil \\ Email: nogueira.g@pucpr.br, escheeren@gmail.com, kruegereddy@gmail.com,percy.nohama@gmail.com, vera@ceb.unicamp.br
}

Received May 1, 2013; revised June 7, 2013; accepted June 15, 2013

Copyright (C) 2013 Guilherme Nogueira-Neto et al. This is an open access article distributed under the Creative Commons Attribution License, which permits unrestricted use, distribution, and reproduction in any medium, provided the original work is properly cited.

\begin{abstract}
Mechanomyography (MMG) acquires the oscillatory waves of contracting muscles. Electromyography (EMG) is a tool for monitoring muscle overall electrical activity. During muscle contractions, both techniques can investigate the changes that occur in the muscle properties. EMG and MMG parameters have been used for detecting muscle fatigue with diverse test protocols, sensors and filtering. Depending on the analysis window length (WLA), monitoring physiological events could be compromised due to imprecision in the determination of parameters. Therefore, this study investigated the influence of WLA variation on different MMG and EMG parameters during submaximal isometric contractions monitoring MMG and EMG parameters. Ten male volunteers performed isometric contractions of elbow joint. Triaxial accelerometer-based MMG sensor and EMG electrodes were positioned on the biceps brachii muscle belly. Torque was monitored with a load cell. Volunteers remained seated with hip and elbow joint at angles of $110^{\circ}$ and $90^{\circ}$, respectively. The protocol consisted in maintaining torque at $70 \%$ of maximum voluntary contraction as long as they could. Parameter data of EMG and the modulus of MMG were determined for four segments of the signal. Statistical analysis consisted of analyses of variance and Fisher's least square differences post-hoc test. Also, Pearson's correlation was calculated to determine whether parameters that monitor similar physiological events would have strong correlation. The modulus of MMG mean power frequency (MPF) and the number of crossings in the baseline could detect changes between fresh and fatigued muscle with 1.0 s WLA. MPF and the skewness of the spectrum $(\mu 3)$, parameters related to the compression of the spectrum, behaved differently when monitored with a triaxial MMG sensor. The EMG results show that for the $1.0 \mathrm{~s}$ and $2.0 \mathrm{~s}$ WLAs have normalized RMS difference with fatigued muscle and that there was strong correlation between parameters of different domains.
\end{abstract}

Keywords: Mechanomyography; Electromyography; Window Length Analysis; Local Muscle Fatigue

\section{Introduction}

During fatiguing muscle contractions, a reduction in maximum voluntary contraction (MVC) occurs due to the inability of myofibrils to produce more force [1] and to the reduction in muscle contraction velocity as well [2]. Electromyography (EMG) has been a useful tool for studying muscle overall electrical activity, function and fatigue [3]. Alternatively, the acquisition of muscle oscillatory response can be useful in monitoring muscle condition [4]. Different types of transducers, from piezo- electric to laser-based distance sensors, can record oscillations non-invasively. Such waves have been measured using accelerometers [5] and the technique is defined as mechanomyography (MMG).

MMG is a non-invasive monitoring technique that can assist in investigating mechanical properties of muscle voluntary contraction and muscle fatigue [6]. Previous studies demonstrated that MMG can provide different information from that obtained with EMG recordings [7], especially muscle fatigue [8], and both could provide 
valuable information about motor unit (MU) recruitment and firing rate [9]. A muscle hypothermia study [10] suggested that the spectral analysis of MMG signals could precisely identify firing rates below the tetanic frequency of activated MUs during muscle contraction, independently of the changes in intrinsic contractile properties. These findings support the idea that $\mathrm{MMG}$ is a reliable method for observing muscle contractile properties in different physiological conditions.

During a task that involves muscle contraction, $\mathrm{MMG}$ time and spectrum characteristics depend on force level and movement rate. From $70 \%$ to $80 \%$ of $\mathrm{MVC}, \mathrm{MMG}$ amplitude [7] and frequency [11] responses of contracting muscles decreased during fatiguing exercises.

There are many studies involving MMG and fatiguing muscle contractions as well as investigations using MMG and EMG simultaneously $[6,8,12,13]$. The use of different MMG methods and sensors can lead to different results. Variations in the analysis window length (WLA) could have influence on torque correlation, on physiological effects or it could compromise the precision of the analysis parameters. A study that monitors signal descriptors varying WLA and muscle physiological condition can help determine which parameter or WLA is more sensitive to detect muscle condition variations. Such parameter and WLA could be useful in control strategies of functional electrical stimulation (FES) systems where muscle fatigue is an important limitation [14].

For EMG, Karlsson et al. [22] used wavelet analysis and determined that WLAs greater than $1.5 \mathrm{~s}$ would be inadequate for monitoring muscle contraction at force levels above $50 \%$ MVC because such intervals would not allow for locally stationary epochs. In this work; however, there was the interest of monitoring $\mathrm{MMG}$ signals from short to long WLAs such as $0.2 \mathrm{~s}$ and $1.0 \mathrm{~s}$, respectively.

Stulen and De Luca built an analog device to track muscle fatigue [23]. They monitored EMG using two parameters: mean power frequency (MPF) and the ratio between the spectral energies comprised in the low and high frequency spectrum regions (delimited by the first MPF calculated with fresh muscle and defined here as spectral ratio-SR). Merletti et al. have been processing [24] and comparing algorithms for estimating EMG activity [25] and they also suggested experiments with different methods for this purpose.

This work aims to observe the influence of WLA on root mean square (RMS) and the mean power frequency (MPF) of MMG and EMG during sustained biceps brachii (BB) muscle submaximal isometric contractions. It is important that torque be monitored with energy-related parameters to assure that EMG and MMG data be acquired at a sustained force level. Another goal is to provide novel results using triaxial accelerometer-based MMG sensors. The reason for studying these parameters is to investigate the influence of varying WLA on their ability to detect changes in muscle condition (specifically, fatigue installation) using the same sensor, protocol and filtering.

\section{Materials \& Methods}

\subsection{Volunteers}

Participated in this study ten physically active male individuals, all college students without neuromuscular disorders or elbow joint problems (age: $22.6 \pm 3.6$ years; weight: $76.5 \pm 9.8 \mathrm{~kg}$; height: $1.80 \pm 0.10 \mathrm{~m})$. The experiments were approved by the Pontifícia Catholic Universidade of Parana's ethics committee (number 2416/08). All participants were informed in detail about the sequence of the test protocol and they agreed to participate giving written informed consent. After skin preparation (trichotomy and cleaning) the volunteer stretched out his elbow joint and the warm up exercise was carried out to avoid muscle damage and consisted of 30 slow dynamic contractions (approximately $50 \%$ s) of the elbow joint with a load of $0.5 \mathrm{~kg}$.

\subsection{Sensors, Electrodes and Adjustable Flat-to-90 ${ }^{\circ}$ Chair}

Figure 1(A) shows the MMG sensor using a Freescale MMA7260Q (USA) triaxial accelerometer with high sensitivity-800 mV/V@1.5 G (G-acceleration of gravity). The sensor provides three acceleration components from which a resultant or overall acceleration can be obtained, termed here as modulus. The complete assemblage weighs about $4 \mathrm{~g}$ (grams) and has dimensions of $14 \mathrm{~mm} \times 16 \mathrm{~mm} \times 2 \mathrm{~mm}$. A separate electronic circuit allowed 10× amplification and $4-40 \mathrm{~Hz}$ Butterworth filtering of $\mathrm{MMG}$ signals, focusing the MMG passband [26]. The sampling frequency was $1 \mathrm{kHz}$ for all signals. No filtering was applied to the torque signal and the EMG was acquired using a commercial device (EMG System do Brasil/Brazil, 1000× differential amplification, 20 - $500 \mathrm{~Hz}$ bandwidth, active bipolar Ag/AgCl Kendall Medi-Trace 100, $\$ 30 \mathrm{~mm}$ foam electrodes/USA). Both the MMG sensor and EMG electrodes were placed on the mid part of the biceps belly on the line between acromion and the fossa cubit, approximately one-third of the distance proximal to fossa cubit [8]. The EMG reference electrode was placed over the olecranon process of ulna. The MMG sensor was equidistantly placed between two active EMG electrodes. The shortest distance that allowed proper operation of the MMG sensor with no mechanical interference from the EMG electrodes was 41 $\mathrm{mm}$. Trichotomy and abrasion of the skin reduced interelectrode impedance.

Figure 1(B) shows the load cell $(100 \mathrm{~kg}, 2.0 \pm 0.1$ 
$\mathrm{mV} / \mathrm{V}$, EMG System do Brasil/Brazil) used for monitor ing torque. It was adapted to a custom-built chair presented in Figure 1(C). A steel chain connected the load cell extremities between the base of the chair and a grip handle. For every participant, the grip handle had to be adjusted to the correct height for the required elbow joint position $\left(90^{\circ}\right)$. The adjustable backrest was reclined so as to keep the hips bent at an angle of $110^{\circ}$.

\subsection{Protocol}

Volunteers performed isometric MVC of the elbow flexors during $5 \mathrm{~s}$. The highest torque value (peak) was used to estimate the $70 \%$ MVC force level, which was taken as reference for the fatigue submaximal test, likewise Vaz et al. [27]. The participants could follow the acquired signals at the target moment of $70 \% \mathrm{MVC}$ on a computer screen. This visual feedback allowed them to check if their torque responses were drifting from the onscreen $70 \%$ MVC reference line thus requiring adjustments in the torque intensity. Throughout the exercise, technicians gave voice commands demanding that volunteers keep the sustained torque level around the reference line for the longest time they could.

\subsection{Data Acquisition}

A LabVIEW ${ }^{\mathrm{TM}}$ program acquired MMG, EMG and torque. All signals and volunteer data were saved into European Data Format (EDF) files. The data acquisition board was a Data Translation ${ }^{\mathrm{TM}}$ DT300 series with a sampling rate of $1 \mathrm{kHz}$.

\subsection{Time Instants, Segments of Signal and Parameters}

The MMG, EMG and torque signals were analyzed at three time instants. Preliminary tests showed that torque values oscillate around the $70 \%$ MVC reference level, showing a high initial peak that should be dismissed. A

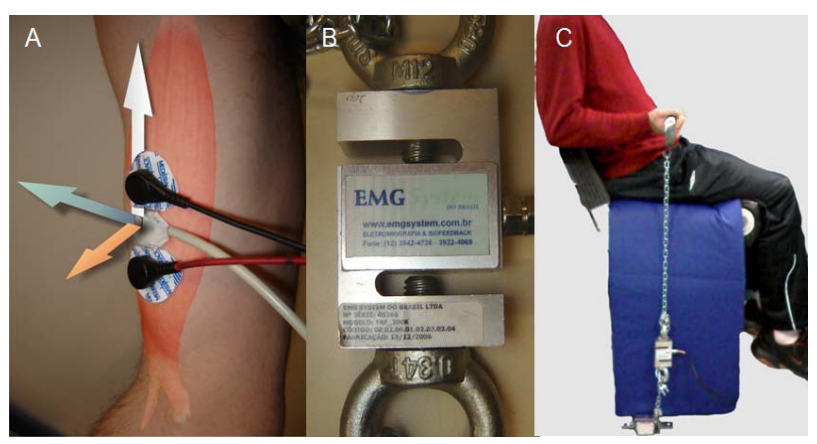

Figure 1. Transducers used in the tests. (A) Mechanomyography sensor with tri-axial accelerometer on biceps brachii muscle belly; (B) load cell, and (C) volunteer seated on custom-built chair. threshold level was determined after each test and defined as the mean value minus two standard deviations extracted from a $5 \mathrm{~s}$ torque epoch in the beginning of the signal, soon after the dismissed initial peak. This threshold level helps determine the beginning and end of the $70 \%$ MVC test, respectively, when torque crosses this level for the first and last times. These limits correspond to the left and right vertical gray lines in Figure 2 where dark boxes that indicate the segments of the signal to be analyzed are also illustrated. All segments in Figure 2 can have variable WLA and are defined as: iniSS - segment that begins at the intersection of the left reference line and torque; finSS - segment that ends at the intersection of the right reference line and torque; midSSthe segment of the signal that is equidistant from iniSS and finSS.

The data were normalized by the values obtained from analyses of MVC level. The RMS and MPF were calculated for all signals, segments and WLAs. The RMS represents the quadratic root mean value. MPF is the frequency that divides the spectrum in two regions of equal energy and is related to the compression that occurs in the spectrum energy while muscles become more fatigued. This characteristic affects the ratio between spectrum regions, spectrum shape and skewness. RMS and MPF are expressed by the Equations (1) and (2).

$$
\begin{aligned}
& R M S=\sqrt{\frac{1}{n}} \sum_{i=1}^{n} x_{i}^{2} \\
& M P F=\frac{\sum_{i=0}^{i=n} f_{i} P\left(f_{i}\right)}{\sum_{i=0}^{i=n} P\left(f_{i}\right)}
\end{aligned}
$$

in the Equations (1) and (2), $x$ is the value of the sample, $n$ is the number of samples in the WLA, $f_{i}$ is the $i^{\text {th }}$ frequency bin, and $P\left(f_{i}\right)$ is the spectral density function.

In addition to lateral displacement, Akataki et al. determined that MMG sensors could monitor changes in the longitudinal shortening of contracting muscles [28]. However, they compared and combined data from two monoaxial sensors placed on different parts of the quadriceps. If muscles vibrate in more than one direction, since this study employs triaxial accelerometer-based MMG sensors, then muscle activity resultant acceleration (the MMG modulus) can be extracted after combining information from the three axes.

First, it is necessary to calculate the parameter values of each axis (X, Y, and $\mathrm{Z}$ ), then, the modulus is computed as shown for the modulus of RMS and MPF in Equations (3) and (4), respectively.

$$
\begin{aligned}
& R M S M o d=\sqrt{R M S_{X}^{2}+R M S_{Y}^{2}+R M S_{Z}^{2}} \\
& R M S M P F=\sqrt{M P F_{X}^{2}+M P F_{Y}^{2}+M P F_{Z}^{2}}
\end{aligned}
$$




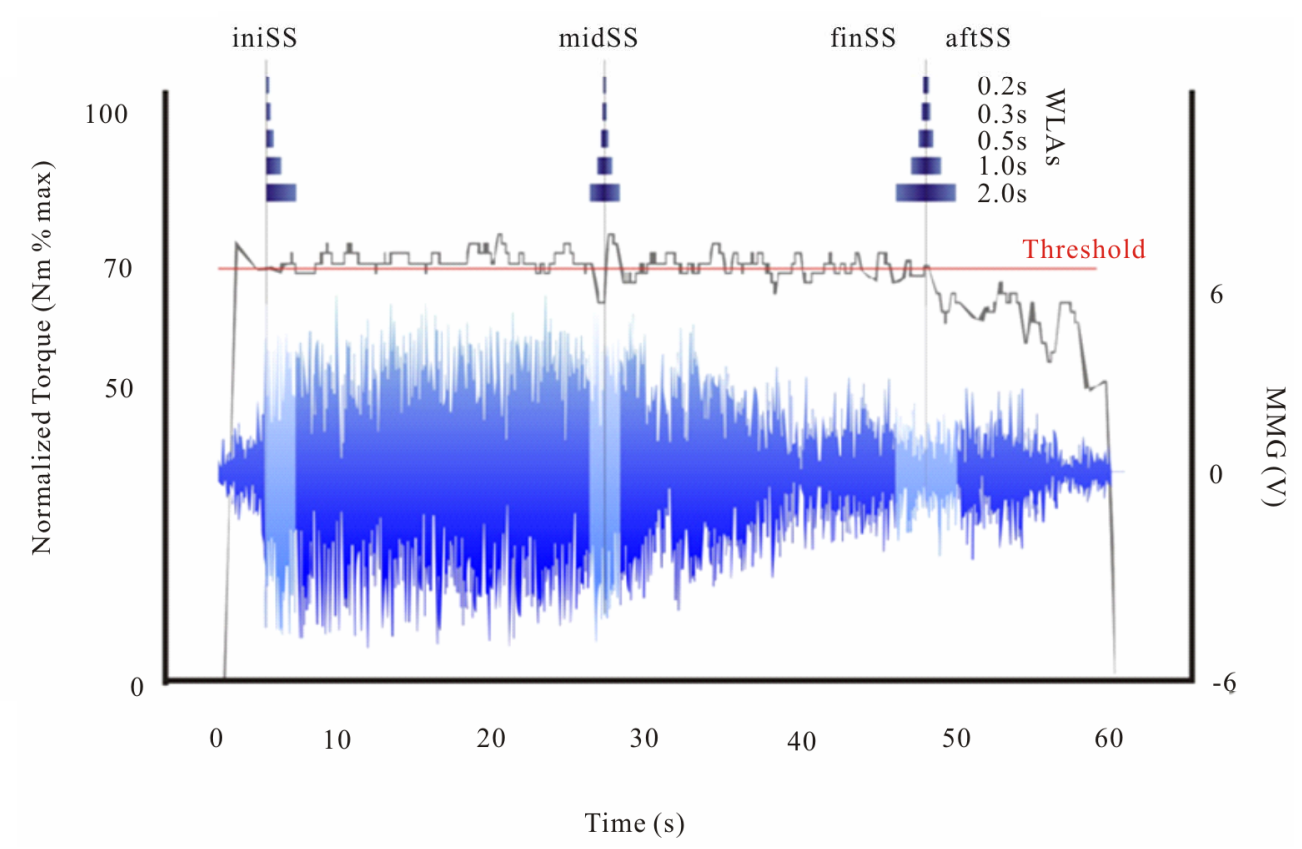

Figure 2. Example of the torque output (gray curve) and mechanomyography (MMG) sensor $Z$ axis (blue curve) for a same subject. Both signals were simultaneously recorded for biceps brachii muscle during sustained submaximal isometric elbow flexion. iniSS, midSS, finSS and aftSS (initial, middle, final, and after signal segments, respectively) appear in the picture with brighter colors. Above the signals are the indication of the analysis window lengths $(2.0 \mathrm{~s}, 1.0 \mathrm{~s}, 0.5 \mathrm{~s}, 0.3 \mathrm{~s}$, and $0.2 \mathrm{~s}$ WLAs) and the threshold is indicated by a red line.

\subsection{Statistical Analysis}

All data are presented as mean \pm standard deviation. Statistical analyses were performed using SPSS ${ }^{\mathrm{TM}}$ for Windows version 11.0. One-way analysis of variance (ANOVA) with Fisher's least square differences (LSD) post hoc test was used to confirm differences between data groups. The first analysis investigated differences between the signal segments (iniSS, midSS and finSS) in every WLA. The second analysis tried to observe statistical differences between WLAs in every signal segment. Since some parameters were supposed to behave similarly, there was the interest in determining whether they would present linear correlation coefficients. Therefore, Pearson's correlation coefficients were calculated. All tests adopted a significance level of $\mathrm{p}<0.05$.

\section{Results}

The average duration of the tests was $46 \pm 16 \mathrm{~s}$. All data were significantly drawn from a normally distributed population according to Shapiro-Wilk tests. The following sections describe the results of ANOVA, Fisher's LSD post hoc and Pearson's correlation tests.

\subsection{Torque}

The parameter investigated in the analysis of torque level was RMS and the results are shown in Table $\mathbf{1}$. Table 1 shows that no statistical differences were found between
iniSS, midSS and finSS. Differences between segments were only found related to aftSS. This implies that volunteers performed the protocol correctly regarding the sustainability of the $70 \%$ MVC torque level from iniSS to finSS. Concerning differences between WLAs, $2.0 \mathrm{~s}$ WLA was different from the others in finSS and aftSS. This can be explained in terms of wide torque oscillations during a $2 \mathrm{~s}$ segment. As initially expected, RMS response behaved similarly to AbsInt. From the inspection of aftSS data, it is noticeable that mean/standard deviation values increase/decrease. This response is in accordance with what can be seen in Figure 2, once bigger WLAs encompass a larger part of the ramp down in the torque signal.

\subsection{Mechanomyography}

Regarding the analyses of MMG signals, only moduli data were considered. Table 3 summarizes mean \pm standard deviation of data that showed statistical differences between either segments or WLAs. Each pair of symbols $(\dagger, \mathrm{x})$ represents data that were different in a comparison between two WLAs. These symbols must be interpreted by looking at a specific segment and parameter (e.g. MPF modulus at finSS). Table 2 indicates that few WLAs presented differences, mainly for $\mu 3$ modulus in aftSS. Few differences were observed between large and short WLAs, specifically with MPF and SR in finSS and $\mu 3$ in midSS. 
Table 1. One-way analysis of variance and Fisher's least squares difference.

\begin{tabular}{|c|c|c|c|c|c|c|c|c|c|c|}
\hline Analysis & iniSS & & midSS & & finSS & & aftSS & & & WLA (s) \\
\hline \multirow{5}{*}{ RMS } & $1.00 \pm 0.00$ & $\ddagger$ & $0.98 \pm 0.04$ & $t$ & $0.94 \pm 0.05$ & $x \neq$ & $0.75 \pm 0.23$ & $\mathrm{x}$ & $\mathrm{c}$ & 2.00 \\
\hline & $1.00 \pm 0.00$ & $\$$ & $0.97 \pm 0.07$ & $t$ & $1.00 \pm 0.04$ & $\dagger+$ & $0.89 \pm 0.09$ & $\dagger$ & $\mathrm{c}$ & 1.00 \\
\hline & $1.00 \pm 0.00$ & + & $0.96 \pm 0.07$ & $t$ & $1.00 \pm 0.04$ & $\dagger+$ & $0.91 \pm 0.06$ & $\dagger$ & $\mathrm{c}$ & 0.50 \\
\hline & $1.00 \pm 0.00$ & $\ddagger$ & $0.97 \pm 0.07$ & & $1.00 \pm 0.04$ & $\dagger+$ & $0.94 \pm 0.04$ & $\dagger$ & $\mathrm{c}$ & 0.30 \\
\hline & $1.00 \pm 0.00$ & 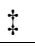 & $0.97 \pm 0.06$ & & $1.00 \pm 0.03$ & $\dagger+$ & $0.96 \pm 0.02$ & $\dagger$ & $\mathrm{c}$ & 0.20 \\
\hline \multirow{5}{*}{ AbsInt } & $1.00 \pm 0.00$ & $\$$ & $0.98 \pm 0.04$ & $t$ & $0.94 \pm 0.06$ & $x \neq$ & $0.74 \pm 0.25$ & $\mathrm{x}$ & $\mathrm{c}$ & 2.00 \\
\hline & $1.00 \pm 0.00$ & $\ddagger$ & $0.97 \pm 0.07$ & $\dagger$ & $1.00 \pm 0.04$ & $\dagger+$ & $0.89 \pm 0.09$ & $\dagger$ & $\mathrm{c}$ & 1.00 \\
\hline & $1.00 \pm 0.00$ & $\$$ & $0.96 \pm 0.07$ & $t$ & $1.00 \pm 0.04$ & $\dagger$ & $0.91 \pm 0.06$ & $\dagger$ & $\mathrm{c}$ & 0.50 \\
\hline & $1.00 \pm 0.00$ & $\ddagger$ & $0.96 \pm 0.07$ & & $0.99 \pm 0.04$ & $\dagger+$ & $0.94 \pm 0.04$ & $\dagger$ & $\mathrm{c}$ & 0.30 \\
\hline & $1.00 \pm 0.00$ & $\$$ & $0.97 \pm 0.06$ & & $1.01 \pm 0.03$ & $\dagger+$ & $0.96 \pm 0.03$ & $\dagger$ & $\mathrm{c}$ & 0.20 \\
\hline
\end{tabular}

Post hoc results of mean \pm standard deviation of normalized torque during iniSS, midSS, finSS and aftSS (initial, middle, final and after signal segments, respectively) and $2.0 \mathrm{~s}, 1.0 \mathrm{~s}, 0.5 \mathrm{~s}, 0.3 \mathrm{~s}$, and $0.2 \mathrm{~s}$ analysis window lengths (WLAs). $\dagger$-value has statistical difference from control (x), $\mathrm{p}<0.05$. $\ddagger$ value has statistical difference from control (c), $\mathrm{p}<0.05$. RMS - root mean square; AbsInt-absolute integral.

Table 2. One-way analysis of variance and Fisher's least squares difference.

\begin{tabular}{|c|c|c|c|c|c|c|c|c|c|c|}
\hline Analysis & iniSS & & & midSs & & finSS & & aftSS & & WLA (s) \\
\hline \multirow{5}{*}{ MPF Modulus } & $1.00 \pm 0.00$ & $\mathrm{c}$ & & $0.96 \pm 0.08$ & \multirow{5}{*}{$\dagger$} & $0.90 \pm 0.08$ & $\pm x$ & $0.91 \pm 0.07$ & $t$ & 2.0 \\
\hline & $1.00 \pm 0.00$ & $\mathrm{c}$ & & $0.90 \pm 0.13$ & & $0.81 \pm 0.11$ & $\dagger$ & $0.85 \pm 0.16$ & + & 1.0 \\
\hline & $1.00 \pm 0.00$ & $\mathrm{c}$ & & $0.93 \pm 0.18$ & & $0.83 \pm 0.14$ & + & $0.81 \pm 0.10$ & $t$ & 0.5 \\
\hline & $1.00 \pm 0.00$ & $\mathrm{c}$ & & $0.87 \pm 0.15$ & & $0.79 \pm 0.12$ & $+\dagger$ & $0.83 \pm 0.15$ & + & 0.3 \\
\hline & $1.00 \pm 0.00$ & $\mathrm{c}$ & & $0.90 \pm 0.19$ & & $0.78 \pm 0.17$ & $\pm \dagger$ & $0.90 \pm 0.17$ & & 0.2 \\
\hline \multirow{5}{*}{$\mu 3$ Modulus } & $1.00 \pm 0.00$ & $\mathrm{c}$ & & $1.05 \pm 0.19$ & \multirow[t]{4}{*}{$\mathrm{x}$} & $1.13 \pm 0.18$ & & $1.08 \pm 0.25$ & $\pm x$ & 2.0 \\
\hline & $1.00 \pm 0.00$ & $\mathrm{c}$ & & $1.10 \pm 0.28$ & & $1.19 \pm 0.36$ & & $1.31 \pm 0.37$ & $+\dagger$ & 1.0 \\
\hline & $1.00 \pm 0.00$ & $t$ & & $1.34 \pm 0.50$ & & $1.38 \pm 0.77$ & + & $1.61 \pm 0.39$ & $\mathrm{c} \dagger$ & 0.5 \\
\hline & $1.00 \pm 0.00$ & & & $1.40 \pm 0.69$ & & $1.50 \pm 0.53$ & & $1.45 \pm 0.41$ & $\dagger$ & 0.3 \\
\hline & $1.00 \pm 0.00$ & & & $1.44 \pm 0.74$ & $\dagger$ & $1.61 \pm 1.10$ & & $1.40 \pm 0.73$ & $\dagger$ & 0.2 \\
\hline \multirow{5}{*}{$\begin{array}{l}\text { Zero-cross } \\
\text { Modulus }\end{array}$} & $1.00 \pm 0.00$ & $\mathrm{c}$ & & $0.89 \pm 0.13$ & \multirow{5}{*}{$\begin{array}{l}+ \\
+ \\
+ \\
+\end{array}$} & $0.81 \pm 0.14$ & $\dagger$ & $0.83 \pm 0.10$ & + & 2.0 \\
\hline & $1.00 \pm 0.00$ & $\mathrm{c}$ & & $0.86 \pm 0.17$ & & $0.77 \pm 0.13$ & $\dagger$ & $0.81 \pm 0.17$ & + & 1.0 \\
\hline & $1.00 \pm 0.00$ & $\mathrm{c}$ & & $0.85 \pm 0.17$ & & $0.73 \pm 0.12$ & + & $0.80 \pm 0.16$ & + & 0.5 \\
\hline & $1.00 \pm 0.00$ & $\mathrm{c}$ & $*$ & $0.86 \pm 0.17$ & & $0.71 \pm 0.16$ & $\pm *$ & $0.81 \pm 0.13$ & + & 0.3 \\
\hline & $1.00 \pm 0.00$ & $\mathrm{c}$ & & $0.86 \pm 0.22$ & & $0.72 \pm 0.18$ & $t$ & $0.82 \pm 0.23$ & & 0.2 \\
\hline \multirow{5}{*}{ SR Modulus } & $1.00 \pm 0.00$ & $\mathrm{c}$ & & $3.32 \pm 5.42$ & \multirow{5}{*}{$t$} & $2.65 \pm 1.98$ & $\dagger$ & $4.11 \pm 3.66$ & + & 2.0 \\
\hline & $1.00 \pm 0.00$ & $\mathrm{c}$ & & $2.90 \pm 2.39$ & & $3.17 \pm 1.71$ & $+\dagger$ & $3.67 \pm 3.71$ & + & 1.0 \\
\hline & $1.00 \pm 0.00$ & $\mathrm{c}$ & & $5.31 \pm 5.83$ & & $4.99 \pm 5.73$ & & $4.66 \pm 5.52$ & & 0.5 \\
\hline & $1.00 \pm 0.00$ & & & $5.67 \pm 7.69$ & & $8.72 \pm 16.1$ & & $20.8 \pm 53.2$ & & 0.3 \\
\hline & $1.00 \pm 0.00$ & $\mathrm{c}$ & & $7.80 \pm 6.69$ & & $14.6 \pm 18.6$ & $\ddagger x$ & $11.1 \pm 20.1$ & & 0.2 \\
\hline
\end{tabular}

Post hoc results of mean \pm standard deviation of normalized mechanomyography (MMG) values during iniSS, midSS, finSS and aftSS (initial, middle, final and after signal segments, respectively) and $2.0 \mathrm{~s}, 1.0 \mathrm{~s}, 0.5 \mathrm{~s}, 0.3 \mathrm{~s}$, and $0.2 \mathrm{~s}$ analysis window lengths (WLAs). $\uparrow$ - value has statistical difference from control (x), $\mathrm{p}<0.05$. $\ddagger, *$ - values have statistical difference from respective controls $(\mathrm{c}, \#), \mathrm{p}<0.05$. MPF-mean power frequency; $\mu 3-$ skewness of the spectrum; zero-crossings - the number of times the biphasic signal crossed the baseline; SR - spectral ratio.

Each pair of symbols $(\ddagger, \mathrm{c})$ represents data that were different in a comparison between two signal segments. These symbols must be interpreted by looking at a specific parameter and two segments (e.g. MPF modulus at iniSS and aftSS). During the sustained submaximal torque exercise performed in this study, for the modulus of MPF, SR and zero-crossing, $1.0 \mathrm{~s}$ WLAs could detect differences between MMG data obtained with fresh and fatigued muscle. With $2.0 \mathrm{~s}$ WLA, SR and $\mu 3$ were un- able to detect such changes. In almost all WLAs, zerocrossing points differences between iniSS and the three other segments, whereas MPF does not differentiate iniSS from midSS. Concerning zero-crossings and MPF, the use of 2.0, 1.0 or $0.5 \mathrm{~s}$ WLAs informed the same differences. $\mu 3$ modulus and SR modulus raised the same differences between iniSS and aftSS with 2.0, 1.0 and 0.5 s WLAs. Although monitoring signals with each of these parameters will investigate the same phenomena (spec- 
trum compression towards the lower frequency region), the use of triaxial MMG modulus response indicates that they can present different results.

\subsection{Electromyography}

Figure 3 shows the normalized EMG RMS (mean \pm standard deviation) of all WLAs and segments. The values of iniSS, midSS and finSS presented statistical difference with aftSS for $2.0 \mathrm{~s}$ WLA. The aftSS presented difference between $2.0 \mathrm{~s}$ and $1.0 \mathrm{~s}$ WLAs. Table 3 summarizes mean \pm standard deviation of all parameters that showed statistical differences between segments.

\subsection{Correlations}

Correlation data show which parameters presented proportional variations along the test. Table 4 and Table 5 inform Pearson's correlation coefficients of all parameters that had at least one strong correlation ( $\mathrm{r}>0.75$ in black font) for MMG and EMG, respectively. This work was interested in verifying whether similar parameters would also show linear correlation. Therefore, the focus was set on correlations of amplitude parameters (RMS, AbsMean, AbsInt, peak-to-peak, and zero-crossing) and spectral parameters (MPF, $\mu 3$ and SR).

Table 4 shows for MMG signal that AbsInt had a strong positive correlation with AbsMean. Interestingly, likewise RMS, none of these parameters showed significant statistical differences between signal segments as indicated in Table 2. MPF showed strong correlation with SR, zero crossing and peak count except with $2.0 \mathrm{~s}$ WLA.
Similarly, peak to peak showed a strong correlation with number of zero-crossings. Nevertheless, zero-cross presented statistical differences between segments as shown in Table 2.

Table 5 shows that EMG RMS had strong correlation with AbsInt. MPF (frequency domain) had a strong correlation with zero-cross (time domain, but related to the dominant frequency), except with $2.0 \mathrm{~s}$ WLA. MPF also showed a strong correlation with SR and $1.0 \mathrm{~s}$ WLA. Both these findings are in accordance with Table 3.

The parameters described in the Methods that are not present in Table 4 and Table 5 did not show statistical differences or strong correlation with other parameters.

\section{Discussion}

Studies conducted in the last decades that analyzed muscle behavior with EMG and MMG techniques used different signal analysis algorithms (Table 6) and different muscle contractions [15,28,29]. Kaplanis et al. conducted a study with many EMG parameters monitored concomitantly [30]. In addition to EMG, this work incorporated triaxial $\mathrm{MMG}$ analysis and aimed to contribute in two ways. First one was to observe the responses of variable-size WLAs of MMG and EMG parameters simultaneously during submaximal fatiguing contractions. The second goal was to use triaxial MMG sensors and to investigate which information would result from the combination of each axis acceleration data. This characteristic can be useful since muscles have a nonuniform distribution of fiber types [31] and geometry can influence muscle response [32]. Individual axes could present interesting results; however, our focus was in response of

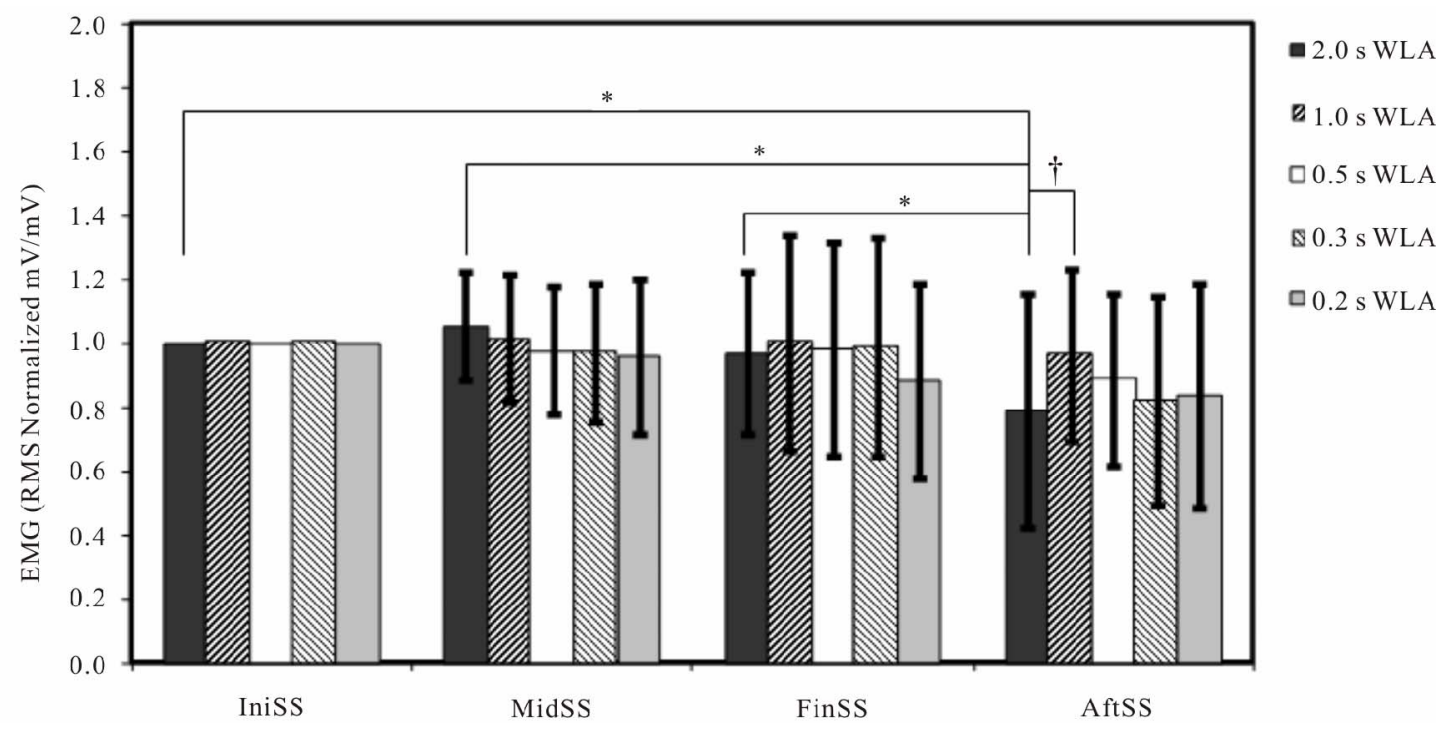

Figure 3. One-way analysis of variance and Fisher's least squares difference post hoc results of electromyography (EMG) normalized root mean square (RMS) values during iniSS, midSS, finSS and aftSS (initial, middle, final and after signal segments, respectively) and their different analysis window lengths (WLAs) - ${ }^{*}$ Significant difference between segments ( $p<$ $0.05){ }^{\dagger}$ Significant difference between $2.0 \mathrm{~s}$ and $1.0 \mathrm{~s}$ WLA $(p<0.05)$. 
Table 3. One-way analysis of variance and Fisher's least squares difference.

\begin{tabular}{|c|c|c|c|c|c|c|c|c|c|c|c|c|}
\hline Analysis & iniSS & & & midSS & & & finSS & & & aftSS & & WLA (s) \\
\hline \multirow{5}{*}{ AbsInt } & $1.00 \pm 0.00$ & & & $1.05 \pm 0.16$ & \multirow{5}{*}{\multicolumn{2}{|c|}{$t$}} & $0.94 \pm 0.27$ & & & $0.78 \pm 0.39$ & \multirow[t]{5}{*}{$\mathrm{c}$} & 2.0 \\
\hline & $1.00 \pm 0.00$ & & & $1.01 \pm 0.15$ & & & $0.97 \pm 0.30$ & & & $0.96 \pm 0.30$ & & 1.0 \\
\hline & $1.00 \pm 0.00$ & & & $0.98 \pm 0.18$ & & & $0.96 \pm 0.29$ & & & $0.88 \pm 0.30$ & & 0.5 \\
\hline & $1.00 \pm 0.00$ & & & $0.96 \pm 0.18$ & & & $0.96 \pm 0.31$ & & & $0.84 \pm 0.3$ & & 0.3 \\
\hline & $1.00 \pm 0.00$ & & & $0.93 \pm 0.22$ & & & $0.90 \pm 0.33$ & & & $0.85 \pm 0.37$ & & 0.2 \\
\hline \multirow{5}{*}{ MPF } & $1.00 \pm 0.00$ & $\mathrm{c}$ & $*$ & $0.90 \pm 0.07$ & \multirow[t]{5}{*}{$\ddagger$} & \# & $0.77 \pm 0.08$ & $\ddagger$ & \multirow{5}{*}{$\begin{array}{l}\text { * } \\
* \\
*\end{array}$} & $0.75 \pm 0.08$ & $\$ *$ & 2.0 \\
\hline & $1.00 \pm 0.00$ & $\mathrm{c}$ & & $0.91 \pm 0.09$ & & \# & $0.77 \pm 0.14$ & $\$$ & & $0.75 \pm 0.14$ & $\$ *$ & 1.0 \\
\hline & $1.00 \pm 0.00$ & $\mathrm{c}$ & & $0.87 \pm 0.12$ & & \# & $0.72 \pm 0.17$ & 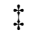 & & $0.76 \pm 0.21$ & $\ddagger$ & 0.5 \\
\hline & $1.00 \pm 0.00$ & $\mathrm{c}$ & & $0.88 \pm 0.15$ & & & $0.78 \pm 0.29$ & $\ddagger$ & & $0.79 \pm 0.24$ & \pm & 0.3 \\
\hline & $1.00 \pm 0.00$ & $\mathrm{c}$ & & $0.85 \pm 0.12$ & & & $0.78 \pm 0.25$ & 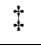 & & $0.72 \pm 0.21$ & $\ddagger$ & 0.2 \\
\hline \multirow{5}{*}{$\mu 3$} & $1.00 \pm 0.00$ & & & $2.80 \pm 4.96$ & & & $4.40 \pm 12.5$ & \multirow{5}{*}{+} & \multirow{5}{*}{ * } & $13.6 \pm 28.9$ & & 2.0 \\
\hline & $1.00 \pm 0.00$ & $\mathrm{c}$ & & $1.08 \pm 0.30$ & & \# & $1.52 \pm 0.73$ & & & $1.44 \pm 0.49$ & $\$$ & 1.0 \\
\hline & $1.00 \pm 0.00$ & $\mathrm{c}$ & & $1.12 \pm 0.36$ & & & $1.22 \pm 0.46$ & & & $1.66 \pm 1.11$ & $\$$ & 0.5 \\
\hline & $1.00 \pm 0.00$ & & & $1.14 \pm 0.47$ & & & $1.31 \pm 0.70$ & & & $1.52 \pm 1.12$ & & 0.3 \\
\hline & $1.00 \pm 0.00$ & & & $1.19 \pm 0.47$ & & & $1.37 \pm 0.81$ & & & $1.57 \pm 1.24$ & & 0.2 \\
\hline \multirow{5}{*}{ Zero-cross } & $1.00 \pm 0.00$ & & & $0.87 \pm 0.11$ & & & $0.80 \pm 0.21$ & \multirow{2}{*}{\multicolumn{2}{|c|}{+}} & $0.89 \pm 0.38$ & \multirow[b]{2}{*}{$\$$} & 2.0 \\
\hline & $1.00 \pm 0.00$ & $\mathrm{c}$ & & $0.87 \pm 0.12$ & & & $0.81 \pm 0.21$ & & & $0.77 \pm 0.23$ & & 1.0 \\
\hline & $1.00 \pm 0.00$ & $\mathrm{c}$ & & $0.87 \pm 0.13$ & & & $0.78 \pm 0.20$ & \multicolumn{2}{|l|}{ t } & $0.78 \pm 0.25$ & $\$$ & 0.5 \\
\hline & $1.00 \pm 0.00$ & $\mathrm{c}$ & & $0.89 \pm 0.13$ & & & $0.77 \pm 0.26$ & \multicolumn{2}{|l|}{$\$$} & $0.80 \pm 0.29$ & \multirow[t]{2}{*}{$\$$} & 0.3 \\
\hline & $1.00 \pm 0.00$ & $\mathrm{c}$ & & $0.93 \pm 0.15$ & & & $0.80 \pm 0.30$ & + & & $0.82 \pm 0.26$ & & 0.2 \\
\hline \multirow{5}{*}{ SR } & $1.00 \pm 0.00$ & $\mathrm{c}$ & & $2.10 \pm 1.60$ & \multirow{3}{*}{\multicolumn{2}{|c|}{ \# }} & $3.60 \pm 3.10$ & \multicolumn{2}{|l|}{$t$} & $3.50 \pm 1.65$ & $t$ & 2.0 \\
\hline & $1.00 \pm 0.00$ & $\mathrm{c}$ & & $1.50 \pm 0.83$ & & & $3.05 \pm 2.39$ & 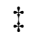 & * & $3.60 \pm 2.07$ & $\$ *$ & 1.0 \\
\hline & $1.00 \pm 0.00$ & $\mathrm{c}$ & & $2.02 \pm 2.03$ & & & $5.75 \pm 7.35$ & \multirow{3}{*}{\multicolumn{2}{|c|}{$\mathrm{c}$}} & $6.08 \pm 7.98$ & \multirow[t]{2}{*}{$\$$} & 0.5 \\
\hline & $1.00 \pm 0.00$ & $\$$ & & $1.39 \pm 0.83$ & 4 & & $4.57 \pm 4.83$ & & & $3.48 \pm 3.01$ & & 0.3 \\
\hline & $1.00 \pm 0.00$ & $\$$ & & $2.15 \pm 2.04$ & + & & $4.28 \pm 3.59$ & & & $8.12 \pm 6.77$ & $\mathrm{c}$ & 0.2 \\
\hline
\end{tabular}

Post hoc results of mean \pm standard deviation of normalized electromyography (EMG) values during iniSS, midSS, finSS and aftSS (initial, middle, final and after signal segments, respectively) and $2.0 \mathrm{~s}, 1.0 \mathrm{~s}, 0.5 \mathrm{~s}, 0.3 \mathrm{~s}$, and $0.2 \mathrm{~s}$ analysis window lengths (WLAs). $\$$, * values have statistical difference from respective controls (c, \#), p < 0.05. iEMG - integral of EMG signal; MPF - mean power frequency; $\mu 3$ - skewness of the spectrum; Zero-cross-the number of times the biphasic signal crossed the baseline; $\mathrm{SR}$ - spectral ratio.

MMG modulus parameters.

Inspecting the literature related to MMG end EMG analysis, it is clear for a preference in the use of $1.0 \mathrm{~s}$ WLA. The analysis of muscle fatigue in isometric muscle contractions requires that EMG signals be wide-sense stationary for segments ranging from $0.5 \mathrm{~s}$ to $2.0 \mathrm{~s}$ WLA at low contraction levels [33], and $0.5 \mathrm{~s}$ to $1.5 \mathrm{~s}$ WLA for $50 \%-80 \%$ MVC [34]. Notwithstanding, there are studies using WLAs ranging from $0.1 \mathrm{~s}$ to $2.0 \mathrm{~s}$.

The volunteers performed sustained $70 \% \mathrm{MVC}$ as long as they could. Table 1 shows data similarity from iniSS to finSS and no significant difference was observed. This implies that volunteers maintained torque statistically around $70 \%$ MVC between iniSS and finSS. In addition, the visual feedback system allowed maintenance of torque level along the $70 \%$ reference line. Although volunteers were instructed to maintain a constant torque, brief MU-generated fluctuations in the torque signal can be explained by the recruitment and derecruitment of MU that are the main mechanisms for generating force between $40 \%$ and $80 \%$ MVC [35]. The 70\% MVC gen- erated in the beginning of the test was determined for a fresh muscle. FinSS is the last signal segment in which the volunteer could keep $70 \% \mathrm{MVC}$ and his muscle was fatigued. In this moment, the torque was the maximum that the participant could generate. We assumed that muscle contractile properties suffered changes during finSS when compared to the muscle condition in iniSS. This assumption is supported by the literature [36] and by statistical differences between the segments in Table 2 for MMG and in Table 3 for EMG.

Some papers used three contractions in order to determine MVC, but in this study volunteers performed only one contraction to quantify the maximum torque. Therefore it is possible that small errors in MVC determination can have occurred. Although care was taken so as to guarantee that volunteers did not perform prohibited movements during the test and the determined $70 \%$ MVC level was successfully sustained from iniSS to finSS, small muscle length changes can have occurred in between. The BB is a biarticular muscle, and the results can have been affected due to small displacements of the 
Table 4. Pearson's correlation between mechanomyography parameters.

\begin{tabular}{|c|c|c|c|c|c|c|c|}
\hline Parameters & WLA & AbsInt & AbsMean & MPF & Peak to Peak & Zero-cross & SR \\
\hline \multirow{5}{*}{ AbsInt } & 2.0 & 1.000 & & & & & \\
\hline & 1.0 & 1.000 & & & & & \\
\hline & 0.5 & 1.000 & & & & & \\
\hline & 0.3 & 1.000 & & & & & \\
\hline & 0.2 & 1.000 & & & & & \\
\hline \multirow{5}{*}{ AbsMean } & 2.0 & 0.999 & 1.000 & & & & \\
\hline & 1.0 & 0.999 & 1.000 & & & & \\
\hline & 0.5 & 0.999 & 1.000 & & & & \\
\hline & 0.3 & 0.999 & 1.000 & & & & \\
\hline & 0.2 & 0.999 & 1.000 & & & & \\
\hline \multirow{5}{*}{ MPF } & 2.0 & 0.159 & 0.159 & 1.000 & & & \\
\hline & 1.0 & -0.556 & -0.556 & 1.000 & & & \\
\hline & 0.5 & -0.602 & -0.602 & 1.000 & & & \\
\hline & 0.3 & -0.610 & -0.611 & 1.000 & & & \\
\hline & 0.2 & -0.592 & -0.591 & 1.000 & & & \\
\hline \multirow{5}{*}{ Peak to Peak } & 2.0 & -0.522 & -0.522 & 0.384 & 1.000 & & \\
\hline & 1.0 & -0.677 & -0.677 & 0.925 & 1.000 & & \\
\hline & 0.5 & -0.614 & -0.614 & 0.759 & 1.000 & & \\
\hline & 0.3 & -0.523 & -0.525 & 0.893 & 1.000 & & \\
\hline & 0.2 & -0.524 & -0.523 & 0.869 & 1.000 & & \\
\hline \multirow{5}{*}{ Zero-cross } & 2.0 & -0.539 & -0.539 & 0.476 & 0.942 & 1.000 & \\
\hline & 1.0 & -0.736 & -0.736 & 0.866 & 0.920 & 1.000 & \\
\hline & 0.5 & -0.686 & -0.686 & 0.808 & 0.874 & 1.000 & \\
\hline & 0.3 & -0.648 & -0.648 & 0.798 & 0.680 & 1.000 & \\
\hline & 0.2 & -0.522 & -0.520 & 0.859 & 0.880 & 1.000 & \\
\hline \multirow{5}{*}{ SR } & 2.0 & 0.210 & 0.210 & -0.535 & -0.596 & -0.561 & 1.000 \\
\hline & 1.0 & 0.757 & 0.757 & -0.798 & -0.835 & -0.760 & 1.000 \\
\hline & 0.5 & 0.680 & 0.680 & -0.677 & -0.644 & -0.696 & 1.000 \\
\hline & 0.3 & 0.486 & 0.486 & -0.489 & -0.420 & -0.387 & 1.000 \\
\hline & 0.2 & 0.405 & 0.405 & -0.636 & -0.550 & -0.597 & 1.000 \\
\hline
\end{tabular}

Data were split by analysis window length (WLA). AbsInt—absolute integral; AbsMean — absolute mean; SR — spectral ratio; MPF—mean power frequency; RMS — root mean square; zero-cross - number of zero-crossings in the segment; peak count—number of peaks in the segment. Although some parameters show strong correlation $(\mathrm{r}<0.75, \mathrm{p}<0.05)$, they did not present significant statistical difference as shown in Table 2.

shoulder joint.

Table 2 indicates that MPF showed differences between the analyzed signal segments. Depending on the WLA, MPF parameters should suffer the negative effect of spectral energy leakage due to the nature of the discrete FFT algorithm [37]. The smaller the number of samples provided to the FFT the greater the resolution loss in the spectrum that will affect the precision of MPF.

The increasing in $\mu 3$ values for MMG signals repressented a spectrum compression to the left that can be confirmed by the decreasing values of MPF. The compres- sion of the spectral content to the lower frequency region means that muscles are becoming fatigued [11].

Table 3 presented EMG data and showed that WLAs of intermediate sizes had more statistical differences between segments than the largest and the smallest WLAs. Since the $70 \%$ MVC level limits the length of WLAs to $1.5 \mathrm{~s}$ [34], differences found with $2.0 \mathrm{~s}$ WLA must be rejected. The WLA that showed more statistical differences was $1.0 \mathrm{~s}$ WLA. However, care must be taken when choosing WLA and force contraction level due to non-stationarity problems. During muscle fatiguing con- 
Table 5. Pearson's correlation between electromyography parameters.

\begin{tabular}{|c|c|c|c|c|c|c|c|c|}
\hline Parameters & WLA & RMS & AbsInt & MPF & $\mu 3$ & Peak-to-Peak & Zero-cross & SR \\
\hline \multirow{5}{*}{ RMS } & 2.0 & 1.000 & & & & & & \\
\hline & 1.0 & 1.000 & & & & & & \\
\hline & 0.5 & 1.000 & & & & & & \\
\hline & 0.3 & 1.000 & & & & & & \\
\hline & 0.2 & 1.000 & & & & & & \\
\hline \multirow{5}{*}{ AbsInt } & 2.0 & 0.985 & 1.000 & & & & & \\
\hline & 1.0 & 0.958 & 1.000 & & & & & \\
\hline & 0.5 & 0.967 & 1.000 & & & & & \\
\hline & 0.3 & 0.965 & 1.000 & & & & & \\
\hline & 0.2 & 0.988 & 1.000 & & & & & \\
\hline \multirow{5}{*}{ MPF } & 2.0 & 0.247 & 0.276 & 1.000 & & & & \\
\hline & 1.0 & -0.104 & -0.107 & 1.000 & & & & \\
\hline & 0.5 & -0.028 & -0.031 & 1.000 & & & & \\
\hline & 0.3 & 0.074 & -0.033 & 1.000 & & & & \\
\hline & 0.2 & 0.068 & 0.061 & 1.000 & & & & \\
\hline \multirow{5}{*}{$\mu 3$} & 2.0 & -0.028 & -0.064 & -0.379 & 1.000 & & & \\
\hline & 1.0 & -0.520 & -0.436 & -0.542 & 1.000 & & & \\
\hline & 0.5 & -0.542 & -0.579 & -0.498 & 1.000 & & & \\
\hline & 0.3 & -0.600 & -0.569 & -0.608 & 1.000 & & & \\
\hline & 0.2 & -0.524 & -0.529 & -0.629 & 1.000 & & & \\
\hline \multirow{5}{*}{ Peak-to-Peak } & 2.0 & 0.677 & 0.660 & 0.358 & 0.091 & 1.000 & & \\
\hline & 1.0 & 0.377 & 0.336 & 0.351 & -0.843 & 1.000 & & \\
\hline & 0.5 & 0.300 & 0.253 & 0.529 & -0.385 & 1.000 & & \\
\hline & 0.3 & 0.497 & 0.474 & 0.449 & -0.339 & 1.000 & & \\
\hline & 0.2 & 0.445 & 0.446 & 0.451 & -0.534 & 1.000 & & \\
\hline \multirow{5}{*}{ Zero-cross } & 2.0 & -0.433 & -0.463 & 0.369 & -0.470 & -0.373 & 1.000 & \\
\hline & 1.0 & -0.201 & -0.252 & 0.853 & -0.302 & 0.196 & 1.000 & \\
\hline & 0.5 & -0.064 & -0.074 & 0.951 & -0.506 & 0.390 & 1.000 & \\
\hline & 0.3 & 0.061 & -0.005 & 0.951 & -0.630 & 0.383 & 1.000 & \\
\hline & 0.2 & 0.001 & 0.000 & 0.893 & -0.553 & 0.360 & 1.000 & \\
\hline \multirow{5}{*}{ SR } & 2.0 & -0.277 & -0.318 & -0.723 & 0.811 & -0.200 & -0.302 & 1.000 \\
\hline & 1.0 & 0.072 & 0.083 & -0.892 & 0.469 & -0.302 & -0.780 & 1.000 \\
\hline & 0.5 & -0.112 & -0.131 & -0.797 & 0.588 & -0.314 & -0.791 & 1.000 \\
\hline & 0.3 & -0.073 & 0.007 & -0.801 & 0.463 & -0.318 & -0.766 & 1.000 \\
\hline & 0.2 & -0.348 & -0.343 & -0.637 & 0.711 & -0.216 & -0.624 & 1.000 \\
\hline
\end{tabular}

Data were split analysis window length (WLA). SR — spectral ratio; AbsInt—integral of rectified EMG signal; RMS—root mean square; MPF — mean power frequency; zero-cross - number of zero-crossings; and $\mu 3$ - skewness of the spectrum.

traction there are a MVC reduction due to the inability of myofibrils to produce force [1] and a reduction in the muscle contraction velocity [2] so that we believe the 1.0 s WLA was the one that better represented EMG pattern variations during the fatiguing protocol, because it could indicate more differences. The EMG interelectrode distance was a limitation in this study, because the Surface Electromyography for the Non-Invasive Assessment of Muscles (SENIAM) project does not recommend using distances above $20 \mathrm{~mm}$. Therefore, unstable recordings with a high level of non-stationarity can have occurred and can be the cause that no differences were observed between WLAs.

The mass of accelerometer-based MMG sensors affects the quality of monitored parameters and excessive mass can cause serious distortions [38]. The sensors were set to operate at the highest allowable sensibility. Therefore, their low weight and small dimensions theoretically favored the acquisition of very small vibrations, conferring more reliability to the MMG acquisition system. On the other hand, it can be argued whether there would be practical benefits in using these sensors because they have high sensibility and, thus, unwanted movements could eventually introduce spurious noise. Indeed, abrupt 


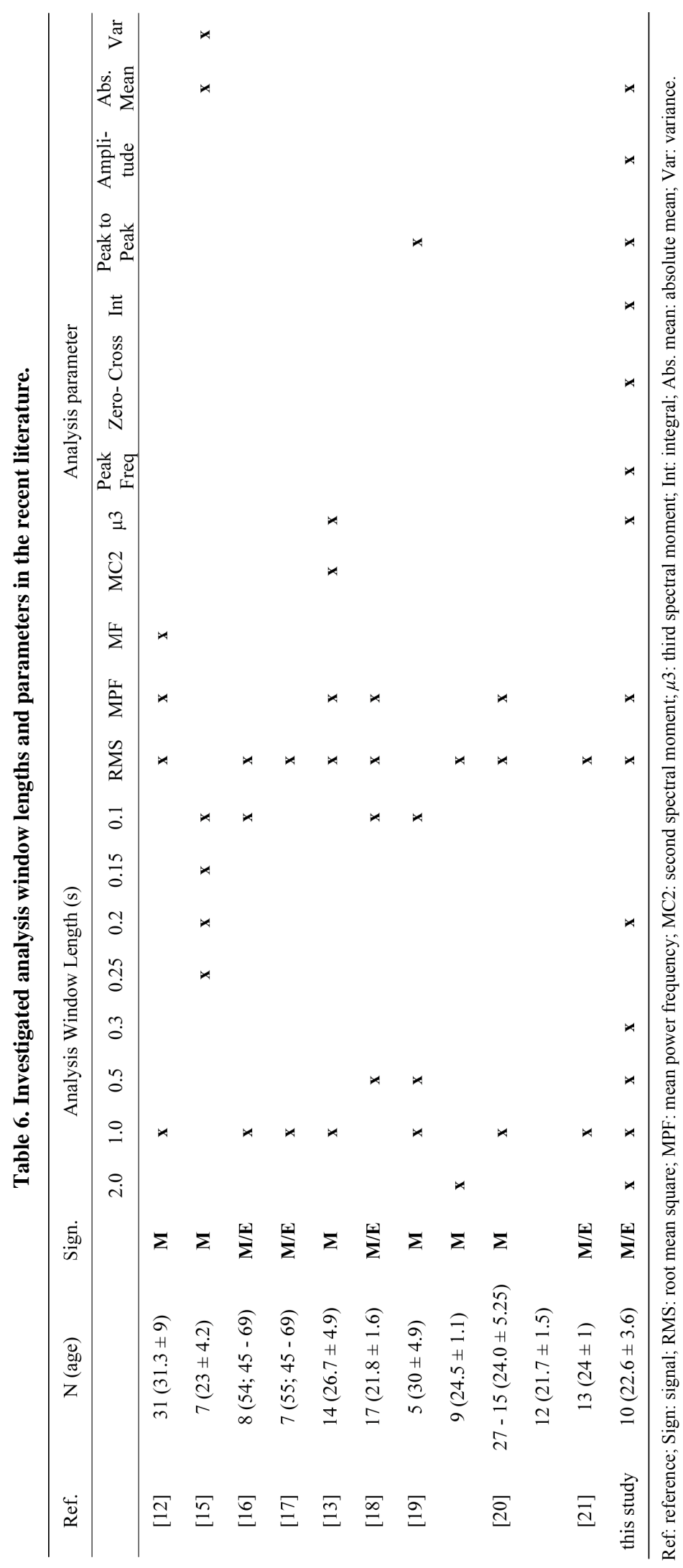


movement artifacts add unwanted noise to the signals and this can be observed even in monoaxial accelerometer-based sensors. Triaxial accelerometers register this abrupt noise in all axes; therefore it can be identified and processed.

The contractions measured in this investigation were under voluntary control. So, some noise has been accepted due to the activity of other muscles involved in the task in addition to BB.

This study investigated many MMG and EMG parameters simultaneously during fatiguing isometric contractions and can help determine useful parameters for neuroprosthesis control strategies. For example, Table 3 shows that MPF presented more differences than SR and $\mu 3$. Zero-crossing analyses identified differences between iniSS and midSS, except for $0.2 \mathrm{~s}$ WLA. In neuroprosthesis control it is important to preview forthcoming losses in torque performance. Zero-crossing and MPF could be used for FES control strategies. In a sustained $70 \% \mathrm{MVC}$ isometric contraction of the BB, first differences could be observed with zero-crossing. Then, when MPF started showing differences between the computed parameters and iniSS, it could be appropriate to start a new FES profile for avoiding most severe effects caused by muscle fatigue in the muscle performance. Apparently, the larger WLAs presented more differences than the smaller ones giving the impression that they are better for monitoring muscle physiological conditions along time or for FES control. However, large WLAs could raise usability problems for real time strategies because decision making based on events that have occurred more than $1 \mathrm{~s}$ before can be impractical or catastrophic. Because of the serious implications, it is recommended that future studies address these problems.

The influence of variations in WLA has been studied for EMG and MMG using temporal and spectral parameters. Regarding MMG, the $1.0 \mathrm{~s}$ WLA had the best tradeoff between WLA and the identification of variations in muscle condition along time. Observing more than one parameter simultaneously can be useful for monitoring muscle fatigue, because they can indicate variations in muscle condition that begin to be observed in different segments and following this response pattern can help detect the installation of severe muscle fatigue. In spite of MPF, $\mu 3$ and SR be related in meaning, MPF and zero-crossing were able to identify more consistent differences between iniSS and the other segments, in spite of variations in WLA. Therefore, they were considered more useful for monitoring changes in muscle condition.

Concerning EMG, the conclusion is that for the aftSS the $1.0 \mathrm{~s}$ and $2.0 \mathrm{~s}$ WLAs have normalized RMS difference. There was strong correlation between different domain parameters (MPF and zero-crossing).
A future step is the investigation of the same parameters and WLAs with different muscles. Because of the amount of data, however, future studies investigating different levels of contraction will concentrate only on few parameters and WLAs.

\section{Acknowledgements}

G. N. N. N., E. M. S., E. K. and P. N. would like to thank $\mathrm{CNPq}$ and CAPES for the financial support and grants received. Authors would also like to thank FINEP, SETI, and Fundação Araucária.

\section{REFERENCES}

[1] K. A. Edman and F. Lou, "Myofibrillar Fatigue versus Failure of Activation during Repetitive Stimulation of Frog Muscle Fibres," Journal of Physiology (London), Vol. 457, 1992, pp. 655-673.

[2] N. A. Curtin and K. A. Edman, "Force-Velocity Relation for Frog Muscle Fibres: Effects of Moderate Fatigue and of Intracellular Acidification," Journal of Physiology (London), Vol. 475, No. 3, 1994, pp. 483-494.

[3] A. Adam and C. J. De Luca, "Recruitment Order of Motor Units in Human Vastus Lateralis Muscle is Maintained during Fatiguing Contractions," Journal of Neurophysiology, Vol. 90, No. 5, 2003, pp. 2919-2927. doi:10.1152/jn.00179.2003

[4] F. V. Brozovich and G. H. Pollack, "Muscle Contraction Generates Discrete Sound Bursts," Biophysical Journal, Vol. 41, No. 1, 1983, pp. 35-40. doi:10.1016/S0006-3495(83)84403-8

[5] C. Orizio, R. Perini and A. Veicsteinas, "Muscular Sound and Force Relationship during Isometric Contraction in Man," European Journal of Applied Physiology, Vol. 58, No. 5, 1989, pp. 528-533. doi:10.1007/BF02330708

[6] M. Gobbo, E. Cè, B. Diemont, F. Esposito and C. Orizio, "Torque and Surface Mechanomyogram Parallel Reduction during Fatiguing Stimulation in Human Muscles," European Journal of Applied Physiology, Vol. 97, No. 1, 2006, pp. 9-15. doi:10.1007/s00421-006-0134-8

[7] C. Orizio, R. Perini and A. Veicsteinas, "Changes of Muscular Sound during Sustained Isometric Contraction up to Exhaustion," Journal of Applied Physiology, Vol. 66, No. 4, 1989, pp. 1593-1598.

[8] K. Søgaard, A. K. Blangsted, L. V. Jørgensen, P. Madeleine and G. Sjøgaard, "Evidence of Long Term Muscle Fatigue Following Prolonged Intermittent Contractions Based on Mechano- and Electromyograms," Journal of Electromyography and Kinesiology, Vol. 13, No. 5, 2003, pp. 441-450. doi:10.1016/S1050-6411(03)00075-0

[9] P. Madeleine, P. Bajaj, K. Søgaard and L. Arendt-Nielsen, "Mechanomyography and Electromyography Force Relationships during Concentric, Isometric and Eccentric contractiOns," Journal of Electromyography and Kinesiology, Vol. 11, No. 2, 2001, pp. 113-121. doi:10.1016/S1050-6411(00)00044-4

[10] T. Kimura, T. Hamada, L. M. Ueno and T. Moritani, 
"Changes in Contractile Properties and Neuromuscular Propagation Evaluated by Simultaneous Mechanomyogram and Electromyogram during Experimentally Induced Hypothermia," Journal of Electromyography and Kinesiology, Vol. 13, No. 5, 2003, pp. 433-440. doi:10.1016/S1050-6411(03)00062-2

[11] C. Orizio, R. Perini, B. Diemont and A. Veicsteinas, "Muscle Sound and Electromyogram Spectrum Analysis during Exhausting Contractions in Man," European Journal of Applied Physiology, Vol. 65, No. 1, 1992, pp. 1-7. doi:10.1007/BF01466266

[12] E. Al-Zahrani, C. Gunasekaran, M. Callaghan, P. Gaydecki, D. Benitez and J. Oldham, "Within-Day and BetweenDays Reliability of Quadriceps Isometric Muscle Fatigue Using Mechanomyography on Healthy Subjects," Journal of Electromyography and Kinesiology, Vol. 19, No. 4, 2008, pp. 695-703. doi:10.1016/j.jelekin.2007.12.007

[13] P. Madeleine, H.-Y. Ge, A. Jaskólska, D. Farina, A. Jaskólski and L. Arendt-Nielsen, "Spectral Moments of Mechanomyographic Signals Recorded with Accelerometer and Microphone during Sustained Fatiguing Contractions," Medical \& Biological Engineering \& Computing, Vol. 44, No. 4, 2006, pp. 290-297. doi:10.1007/s11517-006-0036-2

[14] T. Kesar, L.-W. Chou and S. A. Binder-Macleod, "Effects of Stimulation Frequency versus Pulse Duration Modulation on Muscle Fatigue," Journal of Electromyography and Kinesiology, Vol. 18, No. 4, 2008, pp. 662-671. doi:10.1016/j.jelekin.2007.01.001

[15] N. Alves and T. Chau, "Stationarity Distributions of Mechanomyogram Signals from Isometric Contractions of Extrinsic Hand Muscles during Functional Grasping," Journal of Electromyography and Kinesiology, Vol. 18, No. 3, 2008, pp. 509-515. doi:10.1016/j.jelekin.2006.11.010

[16] P. Vedsted, A. Blangsted, K. Søgaard, C. Orizio and G. Sjøgaard, "Muscle Tissue Oxygenation, Pressure, Electrical, and Mechanical Responses during Dynamic and Static Voluntary Contractions," European Journal of Applied Physiology, Vol. 96, No. 2, 2006, pp. 165-177. doi:10.1007/s00421-004-1216-0

[17] K. Søgaard, C. Orizio and G. Søgaard, "Surface Mechanomyogram Amplitude is not Attenuated by Intramuscular Pressure," European Journal of Applied Physiology, Vol. 96, No. 2, 2006, pp. 178-184. doi:10.1007/s00421-004-1211-5

[18] K. T. Ebersole, K. M. O'Connor and A. P. Wier, "Mechanomyographic and Electromyographic Responses to Repeated Concentric Muscle Actions of the Quadriceps Femoris," Journal of Electromyography and Kinesiology, Vol. 16, No. 2, 2006, pp. 149-157. doi:10.1016/i.jelekin.2005.05.005

[19] Y. Yoshitake, Y. Kawakami, H. Kanehisa and T. Fukunaga, "Surface Mechanomyogram Reflects Length Changes in Fascicles of Human Skeletal Muscles," International Journal of Sport and Health Science, Vol. 3, Special Issue, 2005, pp. 280-285. doi:10.5432/ijshs.3.280

[20] N. Miyamoto and S. Oda, "Effect of Joint Angle on Mechanomyographic Amplitude during Unfused and Fused
Tetani in the Human Biceps Brachii Muscle," European Journal of Applied Physiology, Vol. 95, No. 2-3, 2005, pp. 221-228. doi:10.1007/s00421-005-1359-7

[21] T. T. Matta, T. A. Perini, G. L. Oliveira, J. S. Ornellas, A. A. Louzada, J. Magalhães, L. A. Imbiriba and M. A. Garcia, "Interpretation of the Mechanisms Related to the Muscular Strength Gradation through Accelerometry," Brazilian Journal of Sports Medicine, Vol. 11, No. 5, 2005, pp. 306-310.

[22] S. Karlsson, Y. Jun and M. Akay, "Enhancement of Spectral Analysis of Myoelectric Signals during Static Contractions Using Wavelet Methods," IEEE Transactions on Biomedical Engineering, Vol. 46, No. 6, 1999, pp. 670-684. doi:10.1109/10.764944

[23] F. B. Stulen and C. J. De Luca, "Muscle Fatigue Monitor: A Noninvasive Device for Observing Localized Muscular Fatigue," IEEE Transactions on Biomedical Engineering, Vol. 29, No. 12, 1982, pp. 760-768. doi:10.1109/TBME.1982.324871

[24] R. Merletti and L. R. Lo Conte, "Advances in Processing of Surface Myoelectric Signals: Part 1," Medical \& Biological Engineering \& Computing, Vol. 33, No. 3, 1995, pp. 362-372. doi:10.1007/BF02510518

[25] D. Farina and R. Merletti, "Comparison of Algorithms for Estimation of EMG Variables during Voluntary Isometric Contractions," Journal of Electromyography and Kinesiology, Vol. 10, No. 5, 2000, pp. 337-349. doi:10.1016/S1050-6411(00)00025-0

[26] T. Zagar and D. Krizaj, "Validation of an Accelerometer for Determination of Muscle Belly Radial Displacement," Medical \& Biological Engineering \& Computing, Vol. 43, No. 1, 2005, pp. 78-84. doi:10.1007/BF02345126

[27] M. A. Vaz, Y. T. Zhang, W. Herzog, A. C. Guimaraes and B. R. MacIntosh, "The Behavior of Rectus Femoris and Vastus Lateralis during Fatigue and Recovery: An Electromyographic and Vibromyographic Study," Electromyography and Clinical Neurophysiology, Vol. 36, No. 4, 1996, pp. 221-230.

[28] K. Akataki, K. Mita and Y. Itoh, "Relationship between Mechanomyogram and Force during Voluntary Contractions Reinvestigated Using Spectral Decomposition," European Journal of Applied Physiology, Vol. 80, No. 3, 1999, pp. 173-179. doi:10.1007/s004210050578

[29] M. J. Callaghan, C. J. McCarthy and J. A. Oldham, "The Reliability of Surface Electromyography to Assess Quadriceps Fatigue during Multi Joint Tasks in Healthy and Painful Knees," Journal of Electromyography and Kinesiology, Vol. 19, No. 1, 2009, pp. 172-180. doi:10.1016/j.jelekin.2007.05.004

[30] P. A. Kaplanis, C. S. Pattichis, L. J. Hadjileontiadis and V. C. Roberts, "Surface EMG Analysis on Normal Subjects Based on Isometric Voluntary Contraction," Journal of Electromyography and Kinesiology, Vol. 19, No. 1, 2009, pp. 157-171. doi:10.1016/j.jelekin.2007.03.010

[31] M. A. Johnson, J. Polgar, D. Weightman and D. Appleton, "Data on the Distribution of Fibre Types in Thirty-Six Human Muscles An Autopsy Study," Journal of the Neurological Sciences, Vol. 18, No. 1, 1973, pp. 111-129. doi:10.1016/0022-510X(73)90023-3 
[32] C. J. Zuurbier and P. A. Huijing, "Influence of Muscle Geometry on Shortening Speed of Fibre, Aponeurosis and muscLe," Journal of Biomechanics, Vol. 25, No. 9, 1992, pp. 1017-1026. doi:10.1016/0021-9290(92)90037-2

[33] D. Zazula, S. Karlsson and C. Doncarli, "Advanced Signal Processing Techniques," In: R. Merletti and P. Parker, Eds., Electromyography: Physiology, Engineering, and Noninvasive Applications, Wiley-IEEE Press, New Jersey, 2004.

[34] R. Merletti and M. Knaflitz, "Electrically Evoked Myoelectric Signals," Critical Reviews in Biomedical Engineering, Vol. 19, No. 4, 1992, pp. 293-340.

[35] C. J. De Luca, R. S. LeFever, M. P. McCue and A. P. Xenakis, "Behaviour of Human Motor Units in Different Muscles during Linearly Varying Contractions," The Jour- nal of Physiology, Vol. 329, 1982, pp. 113-128.

[36] B. Bigland-Ritchie, R. Johansson, O. C. Lippold and J. J. Woods, "Contractile Speed and EMG Changes during Fatigue of Sustained Maximal Voluntary Contractions," Journal of Neurophysiology, Vol. 50, No. 1, 1983, pp. 313324.

[37] F. J. Harris, "On the Use of Windows for Harmonic Analysis with the Discrete Fourier Transform," Proceedings of the IEEE, Vol. 66, No. 1, 1978, pp. 51-84. doi:10.1109/PROC.1978.10837

[38] M. Watakabe, K. Mita, K. Akataki and K. Ito, "Reliability of the Mechanomyogram Detected with an Accelerometer during Voluntary Contractions," Medical \& Biological Engineering \& Computing, Vol. 41, No. 2, 2003, pp. 198202. doi:10.1007/BF02344888 